\title{
Progress towards the Global Eradication of Dracunculiasis
}

\author{
Adekunle Sanyaolu*, Chuku Okorie, Yashika Dixon, Muguette Kemajou, Krystel Ly, \\ Elizabeth Mathew, Jerome Nchamukong, Pooyan Nikookar, Roselin Ogunjimi, \\ Risha Patidar, Sonam Sharma, Justin Woei
}

Saint James School of Medicine, Anguilla, BWI

Email: ${ }^{*}$ ksanyaolu@mail.sjsm.org

Received 22 June 2015; accepted 10 July 2015; published 16 July 2015

Copyright (C) 2015 by authors and OALib.

This work is licensed under the Creative Commons Attribution International License (CC BY). http://creativecommons.org/licenses/by/4.0/

\begin{abstract}
Dracunculus medinensis is a parasitic infection that can be prevented but still endemic in few countries today. Most individuals are not even aware that they are infected until when the symptoms develop. Contaminated drinking water, endemicity for the disease, and previous history of infection are the major risk factors for transmission of this disease. The Guinea worm's life-cycle begins in contaminated water and ends within the human body. A host is necessary for the survival of this parasite. Symptoms do not develop until after about one year later. The overall infection process is very painful with a low mortality rate. The duration for treatment may take anywhere from hours, days, or weeks. Management of this parasitic infection is critical and can only be done after the worm has been completely removed. The incidence of the disease has decreased significantly since the 1980s. However, the complete eradication of Dracunculiasis has proven to be a very difficult process. Fortunately, there has been tremendous progress in the steps toward global eradication of the disease. There are several measures, such as surveillance, vector control, health education, provision of portable drinking water and community sensitization and mobilization which are currently being implemented by several governmental and non-governmental organizations in order to contain and hopefully eradicate the disease.
\end{abstract}

\section{Keywords}

Dracunculus medinensis, Eradication, Dracunculiasis, Copepods

Subject Areas: Global Health

\section{Introduction}

Dracunculiasis, also known as Guinea worm disease (GWD) is a parasitic infection that generally begins in an

"Corresponding author.

How to cite this paper: Sanyaolu, A., Okorie, C., Dixon, Y., Kemajou, M., Ly, K., Mathew, E., Nchamukong, J., Nikookar, P., Ogunjimi, R., Patidar, R., Sharma, S. and Woei, J. (2015) Progress towards the Global Eradication of Dracunculiasis. Open Access Library Journal, 2: e1696. http://dx.doi.org/10.4236/oalib.1101696 
individual who has ingested water containing water fleas infected with the guinea worm larvae [1].

Individuals with Dracunculiasis generally do not present with symptoms for about one year after infection [2]. After this period, patients begin to feel ill. Initial symptoms include itchy rash, dizziness, slight fever, nausea, vomiting, and diarrhea [3]. Over time, a blister will develop, most commonly, on the lower extremities around the foot. Over a period of several days, this blister will continue to grow and cause a burning pain. A short period before the worm finally emerges, fever, swelling, and pain may occur in the affected area [2]. Following that, the patient will place the affected body part in cool water to alleviate the pain. When the worm detects the temperature change, it bursts from the blister and releases thousands of larvae into the water to be passed onto the next individual [4]. The infection may cause temporary to permanent disability, affecting the individuals for the rest of their life [1]. In a study done in Imo State, Nigeria, the average duration of symptoms is about 12.7 weeks and about $50 \%$ of all cases of the diseases lead to severe disability [5].

There are many complications that may result from attempting to remove the worm from the body. While the blister is very painful, the pain from removing the worm is much worse. Without proper care, the wound may become infected, which can lead to complications such as formation of abscesses, sepsis, and joint infections that cause deformations, tetanus, and cellulitis [6]. Cellulitis usually occurs in patients with multiple worms and farmers or others that need to continuously stand or walk in their livelihood [7]. The process of removing the worm must be done with care and patience because if the worm breaks during expulsion, it will cause severe inflammation as the dead worm left in the body will begin to degrade [8]. It may also lead to chronic, disabling pain as the worm becomes calcified and a capsule forms around it [7]. This will only lead to additional pain, inflammation, edema, and cellulitis.

The actual death rate of Dracunculiasis is fairly low but disability is a very prominent result. Complications may include ectopic calcification, eosinophilia, pruritus, and skin ulceration [7]. Many individuals have difficult time walking because of pain and further complications caused by secondary bacterial infections. These disabilities generally last about 8 - 9 weeks on average but can be permanent as well. This prevents individuals from working in fields, going to school, taking care of their families, and performing other important tasks. As a result of this, millions of dollars each year are lost due to negative impacts on farming, livestock tending, and the economy [6]. Because Dracunculiasis only occurs in the poorest $10 \%$ of the world's population, the drastic loss in income continues the vicious cycle of disease and poverty.

The clinical presentation of dracunculiasis is so typical, and well known to the local population, hence it does not require laboratory confirmation. In addition, it is found in areas where laboratory services for the diagnosis may be unavailable. While no serologic test is available for the diagnosis of the disease, microscopic examination of the fluid discharged by the worm can show rhabditiform larvae. The appearance of a characteristic "white cloud," representing release of larvae is visible with the naked eye. Diagnosis is obvious once the white, filamentous adult worm appears at the cutaneous ulcer [9] [10].

Over the years, various organizations and corporations have supported the goal of eliminating Dracunculiasis. The Center for Disease Control and Prevention (CDC), The Carter Center (TCC), The United Nations Children's Emergency Fund (UNICEF), and The World Health Organization (WHO) have been assisting ministries of health in Dracunculiasis-endemic countries in meeting the target of eradicating this disease. Recent statistics show tremendous progress towards accomplishing this goal. About 1058 cases were reported in 2011, while in 2012, only 542 cases were reported. Furthermore, there is a significant decrease in the number of cases reported compared with almost 1 million cases in the 1980s, when the initiative was launched [11]. As of 2013, the disease still remains endemic in four countries (Chad, Ethiopia, Mali and South Sudan), but there has been a large decrease in the overall rate of cases compared with the previous year, where 148 cases were reported. Unfortunately, there are still few major challenges to dracunculiasis eradication that are yet to be resolved, especially poor surveillance and containment, unusual epidemiologic patterns, and lack of clean drinking water [12].

\section{Epidemiology and Risk Factors}

It is evident that over decades, the number of cases of Dracunculiasis has decreased dramatically. Table 1 illustrates this decline. In 1986, there were about 3.5 million cases of Dracunculiasis reported in parts of Africa, Asia, and the Middle East. Comparatively, in 2013, 148 cases were reported worldwide, 76\% from South Sudan and the other cases from Chad, Ethiopia, Mali, and Sudan.

The biggest risk factor is drinking water from stagnant ponds, pools in drying riverbeds, and shallow 
Table 1. The dramatic decrease in Dracunculiasis cases over the years.

\begin{tabular}{cccc}
\hline Year & $\mathbf{1 9 8 6}$ & $\mathbf{2 0 1 2}$ & 2013 \\
\hline Cases of Dracunculiasis & 3.5 million & 542 & 148 \\
\hline
\end{tabular}

uncovered wells, contaminated with copepods (tiny "water fleas") that carry Guinea worm larvae. Copepods eat the larvae, after which it takes about 2 weeks to mature, before it can infect humans. Therefore, Dracunculiasis affects poor communities in remote parts of Africa that lack safe drinking water mainly due to the lack of awareness of infectious water sources [13]. Guinea worm incidence may vary based on its proximity to the natural environment or features of the built environment [14].

A seasonal pattern has been noted for Dracunculiasis. Individuals seem to become infected during the rainy season in dry regions, when the stagnant surface is available. However, in wet regions, individuals become infected when water becomes stagnant, or is drying up.

The major determinant for being infected by Dracunculus is the source of water used in different areas and countries. There is an equal distribution of infection between male and female but the prevalence may differ based on location [13]. There is a higher prevalence of the disease among males in India and occasionally in West Africa region, while the prevalence among females is higher in Ethiopia [15]. In addition, infection may occur at any age, but is more common among the young adults 14 - 45 years old. This may be due to the individual's occupation, which may involve working outdoors [12]. Another study that looked at 4 high-endemicity and 23 lower-endemicity villages in South Kordofan, Sudan, noted that prevalence increased with age [15]. In endemic areas, Dracunculiasis most commonly affects those with lower socioeconomic status, who do not have access to protected water sources [16].

Another major risk factor for infection with Dracunculus is previous history of infection. Individuals do not become immune to the infection, and may use the same contaminated water supply year after year thereby predisposing them to the same infection. It may also be due to a slight biological factor that increases the susceptibility in specific individuals [13].

\section{Eradication}

According to the World Health Organization (WHO), the initiative to eradicate Dracunculiasis began in May 1981. Through the years, the organization gained strength through collaboration with the International Commission for the Certification of Dracunculiasis Eradication (ICCDE), WHO, UNICEF, CDC and TCC.

There were about 3.5 million GWD cases worldwide in 1986 and presently, the number of outbreaks has been restricted to poor communities in remote parts of Africa [17]. The disease mainly affects those in rural, deprived, and isolated communities who depend mainly on open stagnant surface water sources, such as ponds, for drinking.

Unfortunately, there is no vaccine or medication for GWD [18]. With the knowledge that the disease is transmitted mainly through consumption of contaminated water, efforts of eradication programs focus mainly on ensuring access to improved drinking water and education on the disease [19]. By the year 2000, Dracunculiasis was already restricted to Africa.

The WHO advocates for eradication, provides technical guidance, coordinates eradication activities, enforces surveillance in Dracunculiasis free areas, monitors and reports on progress achieved. They are the only organization mandated to certify countries as free of the disease following recommendations made by ICCDE. The latest data from the WHO is depicted in Table 2.

In 2014, the annual incidence of the disease had decreased by more than 99\% compared to the mid-1980s [19]. In order to adequately conclude that a country is free of the disease there are various steps to follow.

A country without any new reported cases for 12 consecutive months is believed to have interrupted transmission. This is the pre-certification stage and it must be maintained for at least 3 years since the last indigenous case, during which intense surveillance activities need to continue. ICCDE is made up of 9 public health experts who meet to evaluate the status of transmission in countries applying for certification of Dracunculiasis eradication. It is also their responsibility to recommend whether a particular country should be certified as free of transmission. Even after certification, surveillance should be maintained until global eradication is declared [19]. All these steps and processes for eradication are closely monitored by WHO. 
Table 2. Comparing the incidence of Dracunculiasis over the years.

\begin{tabular}{|c|c|c|}
\hline Latest Data & Comparison & \%decrease \\
\hline 115 cases in January-October 2014 & 139 cases in January-October 2013 & decrease of $\mathbf{1 7 \%}$ \\
\hline 148 total cases reported in 2013 & 542 total cases reported in 2012 & decrease of $\mathbf{7 3 \%}$ \\
\hline $\begin{array}{l}4 \text { endemic countries in } 2014 \\
\text { (Chad, Ethiopia, Mali and South Sudan) }\end{array}$ & 20 endemic countries in 1990 & decrease of $\mathbf{7 5 \%}$ \\
\hline
\end{tabular}

There are still many challenges that prevent complete eradication, including finding and containing the last remaining cases of the disease, which are typically found in remote, often inaccessible, rural areas, According to the CDC, the greatest risk for Dracunculiasis is having a previous infection with the disease because immunity does not exist. WHO also includes animal populations in the measure of successful complete eradication of Dracunculiasis. This unusual disease epidemiology has been reported in the canine population along the river Chari in Chad, one of the four countries left in the battle against the parasitic disease. Despite these setbacks, the WHO is proud of her achievements so far. If global eradication is successful, Dracunculiasis will be the first parasitic disease to be eradicated.

\section{Current Situation}

In 1986 a few years after the WHO initiated the eradication program for Dracunculiasis, there were 3.5 million cases, involving twenty countries. During that time, the original goal was set at $90 \%$ eradication by the year 1991, and for complete eradication of Dracunculiasis by 2009. However, such goal was not achieved. Based on the 2011 health organization survey, the total cases in 2011 was reduced to 1058 cases, which was mainly reported in Mali, South Sudan, Chad, and Ethiopia [20]. It is important to note that during the year 2011, five countries were announced free of the transmission of Dracunculiasis (Bosnia Herzegovina, Brunei Darussalam, Burkina Faso, Eritrea, and Togo). Pakistan became free of the disease in 1993, followed by Sudan in 1995. Eradication in Sudan proved difficult, but due to the concerted efforts of TCC and other organizations in addition to community efforts the eradication was successful. Key to this success, according to TCC, was the work of village volunteers, who educated individuals about the need to filter drinking water. Other countries followed suit, and by 2004, the worm was eradicated in Asia.

Currently, Ghana is winning the battle against the Guinea worm disease as well. According to statistical analysis, there were no reports of disease as at November 2013 as compared with 2008 when 501 new cases were reported, and 2007 when 3357 cases were reported. Majority of this decline can be attributed to the number of interventions in the previous two years, including monitoring dams and other sources of drinking water in endemic communities to ensure infected people do not contaminate the water sources [20].

Additionally, water filters and other water treatments are being provided at both water pumping sites and homes to treat water before drinking. This is due to the efforts of the National Guinea Worm Eradication Program (NGWEP), UNICEF, the Japanese International Cooperation Agency (JAICA), and the European Union (EU) [21].

As shown in Table 3, 2013 marked a year of great progress in total eradication, as 542 cases from 2012 was reduced to 148 cases (73\% reduction). This year marked only four countries that still remain endemic to Dracunculiasis and nine countries that remained to be certified. Data from two countries, Angola and Democratic Republic of Congo, still remained unknown due to internal conflicts. The eradication program of 2014 was successful, as the total number of cases was almost reduced by half ( $71 \%$ decrease in cases with total of 27 confirmed cases). Based on the current reduction rate, it is possible to completely eradicate Dracunculiasis by the year 2017-2018 [22].

Overall, what lessons can be learned during the entire course of Dracunculiasis? While it is difficult, it seems clear that a constant source of funding and logistics will make such eradication programs in the future far more secure, efficient, and economical. For example, the loss of funding in Ghana in 2013 left more people infected, set the campaign back, and led to higher long term costs. The second lesson is the power of community efforts and dedicated community workers who are the real heroes in this long fight against Dracunculiasis. They proved to be quite effective in educating the public and managing cases, and should be trusted in further campaigns. 
Table 3. Number of reported Dracunculiasis cases, by country and local interventions-worldwide, 2012-2013. Adapted from progress in eradication of Dracunculiasis, January 2013-June 2014 [40].

\begin{tabular}{ccccccccc}
\hline Country & 2012 & 2013 & 1-yr change (\%) & Jan.-June 2013 & Jan.-June 2014 & $\begin{array}{c}\text { 6-months } \\
\text { change (\%) }\end{array}$ & Cases (\%) \\
\hline South Sudan & 521 & 113 & $(-78)$ & 74 & 19 & $(-74)$ & 79 \\
Mali & 7 & 11 & $(+57)$ & 4 & 0 & $(-100)$ \\
Chad & 10 & 14 & $(+40)$ & 5 & 6 & $(+20)$ & $(-71)$ & 100 \\
Ethiopia & 4 & 7 & $(+75)$ & 7 & 2 & 0 & $(-100)$ & $\mathbf{( - 7 1 )}$ \\
Sudan & & 3 & & 2 & $\mathbf{2 7}$ & $\mathbf{7 8}$ \\
Total & $\mathbf{5 4 2}$ & $\mathbf{1 4 8}$ & $\mathbf{( - 7 3 )}$ & $\mathbf{9 2}$ & & & \\
\hline
\end{tabular}

\begin{tabular}{|c|c|c|c|c|c|c|c|c|}
\hline \multicolumn{5}{|c|}{ Reported cases } & \multicolumn{4}{|c|}{ Villages under active surveillance in 2013} \\
\hline Country & $\begin{array}{l}\text { Indigenous } \\
\text { in } 2013\end{array}$ & $\begin{array}{c}\text { Imported } \\
\text { in } 2013\end{array}$ & $\begin{array}{c}\text { Contact } \\
\text { during } \\
2013(\%)\end{array}$ & $\begin{array}{l}\text { Change in indigenous } \\
\text { cases in villages under } \\
\text { surveillance } \\
(2012-2013)\end{array}$ & Number & $\begin{array}{l}\text { Reporting } \\
\text { monthly (\%) }\end{array}$ & $\begin{array}{l}\text { Reporting } \\
\text { imported }\end{array}$ & $\begin{array}{l}\text { Reporting } \\
\text { indigenous }\end{array}$ \\
\hline South Sudan & 113 & 0 & 67 & $(-78)$ & 6682 & 100 & 40 & 39 \\
\hline Mali & 11 & 0 & 64 & $(57)$ & 101 & 85 & 0 & 8 \\
\hline Chad & 14 & 0 & 57 & $(40)$ & 703 & 100 & 0 & 9 \\
\hline Ethiopia & 7 & 0 & 57 & (75) & 72 & 93 & 1 & 4 \\
\hline Sudan & 3 & & 100 & & & & & 1 \\
\hline Total & 148 & 0 & 66 & $(-73)$ & 7558 & 99 & 41 & 61 \\
\hline
\end{tabular}

\begin{tabular}{cccccc}
\hline & \multicolumn{5}{c}{ Status interventions in endemic villages in 2013 } \\
\hline Country & $\begin{array}{c}\text { Endemic villages } \\
2012-2013\end{array}$ & $\begin{array}{c}\text { Reporting } \\
\text { monthly }\end{array}$ & $\begin{array}{c}\text { Filters in all households } \\
(\%)\end{array}$ & $\begin{array}{c}\text { Using temephos } \\
\text { Providing health } \\
\text { education }\end{array}$ \\
\hline South Sudan & 106 & 100 & 98 & 96 & 97 \\
Mali & 8 & 75 & 100 & 75 & 75 \\
Chad & 2 & 100 & 100 & 50 & 100 \\
Ethiopia & 2 & 100 & 100 & & 100 \\
Sudan & 0 & & & 94 & 96 \\
Total & 118 & 98 & 98 & & \\
\hline
\end{tabular}

\section{Prevention and Control}

Dracunculiasis transmission can be interrupted at two places in the parasites' life-cycle-by preventing people with open sores from contaminating water sources, and by filtering drinking water [23]. The interventions that are set in place to prevent dracunculiasis focus on the following areas: Surveillance and case containment, provision of safe drinking water, vector control using a chemical larvicide, and health education and community mobilization. Safe drinking water sources include bore-holes and dug wells with covers and springs containing protective walls around them in order to prevent those that are infected with worms from wading and contaminating the water [8]. Development and repair of safe drinking water is of utmost priority along with the filtration of any water source that may be contaminated. Also, heightening surveillance in order to detect the occurrence of each case within 24 hours of worm emergence will aid in containment of disease.

The second point that was implemented to promote prevention is vector control. The vector for guinea worm 
is copepod and in order to control this vector, there is measured amount of approved chemical larvicide, such as abate also known as Temephos; it is an organophosphate larvicide that is added into contaminated water sources to treat water [19]. The chemical agents destroy the copepods thus preventing people from being infected upon consumption of water. Therefore, understanding of the lifecycle of the parasite is important in order to effectively halt the parasite reproductive cycle by using larvicides to kill the cyclops which serve as a host for Guinea worm larvae [24].

It is imperative that we educate communities regarding the disease process and its mode of transmission. Focus should heavily be placed on primary prevention through delivering educational material in form of fliers, pamphlets, and related materials [25]. Preventing those that have the disease from coming in contact with the water source will aid significantly in case containment, hence, educating the population is essential. The community has to undergo behavioral change where they have to imbibe the habit of doing certain things for an extended time period, hence, this altered mind set will aid in further progression towards eradication [26].

An important component in the control of dracunculiasis is surveillance, which serves to detect all cases in a timely manner in such a way that the last case of the disease is known and that there has not been any sign of transmission. There are three different stages a country has to undergo that utilize surveillance. Following disease transmission interruption, the first stage is the endemic stage where nationwide surveillance is used to see if the country is able to show evidence that transmission has not occurred for a sustained amount of time. In the case of dracunculiasis, this means no incidence of the disease for 12 consecutive months [27]. Once the country has been able to present proof of this status, the World Health Organization will allow the country to enter precertification stage where the country is then suitable for certification as dracunculiasis-free. Finally, the last stage is post-certification, where the World Health Organization has deemed a country free of dracunculiasis transmission meaning that surveillance in that country will continue until eradication of dracunculiasis is officially worldwide [28].

Types of surveillance will vary depending on the location. In the case of the disease occurring in a secluded area that is inaccessible to healthcare services, other methods of surveillance are required to detect cases of the disease. In this scenario, there would be searches done from house-to-house to question people in the community to see if a guinea worm case has been seen to appear from anyone by showing an identification card of the nematode [28]. Nowadays, dracunculiasis cases that arise are immediately reported for investigation and containment, and these cases are nationally reported on a monthly basis [14]. The use of incentives has also been utilized to facilitate surveillance for cases that substantiate dracunculiasis. The reward amount awarded for the report of cases and for patients in these countries ranged from US $\$ 40$ to US $\$ 160$ [28].

Another way to control dracunculiasis is to reduce the risk of water-borne diseases using sustainable methods. This can be accomplished by provision of clean drinking water. These sources of potable water include borehole wells, protected deep wells or springs that are enclosed by a wall and cap to prevent disease transmission via water contamination [29]. Studies that were conducted in Africa showed an $81 \%$ reduction in the incidence of dracunculiasis with the usage of borehole water [30]. These wells are manually maintained thereby proper training is required for continued usage. The preferred water source of copepod is shallow and stable surface water which are "man-made ponds", hence it is important to protect groundwater sources by conducting additional surveillance [31].

In cases, where a person has been infected by the guinea worm, it is vital to control the spread of the disease, where certain steps have to be taken in community mobilization. Cases must be identified within 24 hours of the nematode's emergence [32]. During this time, proper management steps have to be taken to reduce breakage by obtaining proper history to determine potentially infected water sources. Proper steps taken to manage cases of dracunculiasis will enable prompt mobilization of the community to institute measures for prevention to avoid contamination of water supplies and spread of the disease [33]. Finally, it is important to ensure that proper treatment has been administered to control the spread of the disease.

\section{Management and Treatment}

Currently, there is neither a vaccine to prevent nor a drug to treat Dracunculiasis. Effective management of the disease focuses on prevention [34]. However, relief from the parasitic infestation may be acquired after complete removal of the worm from the body, using a stick or gauze [8]. Following a known infection with the worm, as well as blistering on the skin, the affected body part needs to be immersed in water in order to hasten 
the emergence of the worm to the body surface [35]. A warm compress may also be used. The contaminated water should be discarded in the soil far away from any drinking water source. Using a stick to wind the worm or a piece of gauze to hold the worm and thus provide some traction, the live worm can be gently, as well as slowly, pulled out of the body [36]. It is advisable to stop pulling the worm whenever any resistance is met in order to avoid breaking the parasite, hence causing putrefaction or petrifaction. During removal, which may last hours, days or weeks, the wound area can be massaged to loosen and ease worm emergence. "Tamale oil" has also been used to lubricate and guide worm extraction [37]. Caution is necessary to ensure the complete extraction of the worm from the body. Wound care with topical antibiotic ointments, such as Bacitracin or Bactroban, after worm removal is helpful in preventing secondary bacterial infection from tetanus. Metronidazole (drug of choice) or Thiabendazole may be used as an adjuvant in worm extraction. Aspirin or ibuprofen is used to treat inflammation and pain from the infection [36]. A surgical extraction of the worm can also be performed prior to blistering and ulceration to the skin [38] [39].

\section{Conclusion}

In conclusion, the incidence of Dracunculiasis has decreased significantly since the initial 3.5 million cases reported in 1986 and only a handful of countries remain that are still endemic to this disease. Although the number of cases has dropped exponentially, complete eradication of Guinea worm disease has not been accomplished. Moving forward, if the reduction rate continues, it is possible to completely eliminate Dracunculiasis by 20172018. The ICCDE has made tremendous efforts to try and eradicate this disease by providing safe drinking water, education on the disease to individuals in the endemic countries, vector control of the parasite's larvae, as well as surveillance and containment. It is also imperative that any case of Guinea worm disease should be identified, reported, contained, and treated before it further spreads. With the continuing efforts of public health organizations in partnership with local communities, Dracunculiasis can be eliminated from the world in the near future.

\section{References}

[1] Centers for Disease Control and Prevention (CDC) (2013) Morbidity and Mortality Weekly Report: Progress toward Global Eradication of Dracunculiasis—January 2012, June 2013. 829-833. 62. No. 42. Retrieve form UMUC Data Base on February 10, 2014.

[2] (2014) Guinea Worm Disease Symptoms, Causes, Treatment-What Are the Signs and Symptoms of Guinea Worm Disease? Medicine Net. http://www.medicinenet.com/guinea_worm_disease/page2.htm

[3] Heiser, L. and Smith, S. (n.d.). Dracunculiasis. http://web.stanford.edu/group/parasites/ParaSites2001/dracunculiasis/DRACUNCULIASIS.html

[4] (2015) Dracunculiasis: Treatment, Symptoms, Advice and Help. http://www.surgerydoor.co.uk/advice/diseases/dracunculiasis/

[5] Smith, G., Blum, D., Huttly, S., Okeke, N., Kirkwood, B. and Feachem, R. (2012) Disability from Dracunculiasis: Effect on Mobility. Annals of Tropical Medicine and Parasitology, 83, 151-158.

[6] Centers for Disease Control and Prevention (CDC) (2012) Parasites_Dracunculiasis (Also Known as Guinea Worm Disease). http://www.cdc.gov/parasites/guineaworm/disease.html

[7] (2015) Complications of Dracunculiasis Guinea Worm Infection. http://www.healthline24x7.com/diseases/infections/dracunculiasis-guinea-worm-infection-/complications.http://www.r ightdiagnosis.com/d/dracunculiasis/complic.htm\#complication_list

[8] Pearson, R. (2013) Dracunculiasis. In: The Merck Manuals-Professional Edition. http://www.merckmanuals.com/professional/infectious_diseases/nematodes_roundworms/dracunculiasis.html\#v10140 $\underline{06}$

[9] Centers for Disease Control and Prevention (CDC) http://www.cdc.gov/dpdx/dracunculiasis/dx.html

[10] (2015) The Carter Center: Dracunculiasis. http://www.cartercenter.org/resources/pdfs/news/health_publications/guinea_worm/AAP-dracunculiasis.pdf

[11] Sergiev, V.P., Migliorini, L., Litvinov, S.K. and Chernikova, E.A. (2014) Neglected Tropical Diseases: A New World Health Organization Program. Meditsinskaia parazitologiia i parazitarnye bolezni, 2, 59-63.

[12] Al-Awadi, A., Al-Kuhlani, A., Breman, J., Doumbo, O., Eberhard, M., Guiguemde, R., Magnussen, P., Molyneux, D. and Nadim, A. (2014) Guinea Worm (Dracunculiasis) Eradication: Update on Progress and Endgame Challenges. 
Transactions of the Royal Society of Tropical Medicine and Hygiene, 108, 249-251. http://dx.doi.org/10.1093/trstmh/tru039

[13] CDC (2015) CDC_-Guinea Worm Disease_Epidemiology \& Risk Factors. http://www.cdc.gov/parasites/guineaworm/epi.html

[14] Royal, N. (2013) Dracunculiasis, Proximity, and Risk: Analyzing the Location of Guinea Worm Disease in a GIS. Transactions in GIS, 17, 298-312. http://dx.doi.org/10.1111/tgis.12003

[15] Cairncross, S., Muller, R. and Zagaria, N. (2002) Dracunculiasis (Guinea Worm Disease) and the Eradication Initiative. Clinical Microbiology Reviews, 15, 223-246. http://dx.doi.org/10.1128/CMR.15.2.223-246.2002

[16] Watts, S. (2000) Dracunculiasis in the Caribbean and South America: A Contribution to the History of Dracunculiasis Eradication. Medical History, 44, 227-250.

[17] CDC (2014) CDC—Guinea Worm Disease-Eradication Program. http://www.cdc.gov/parasites/guineaworm/gwep.html

[18] Barry, M. (2007) The Tail End of Guinea Worm—Global Eradication without a Drug or a Vaccine. New England Journal of Medicine, 356, 2561-2564. http://dx.doi.org/10.1056/NEJMp078089

[19] WHO (2015) WHO_Dracunculiasis (Guinea-Worm Disease). http://www.who.int/dracunculiasis/en/

[20] Centers of Disease Control and prevention, CDC (2011) Morbidity and Mortality Weekly Report (MMWR): Renewed Transmission of Dracunculiasis -Chad, 2010. http://www.cdc.gov/mmwr/preview/mmwrhtml/mm6022a4.htm

[21] Guinea Worm Race 2004 (2005) http://web.stanford.edu/group/parasites/ParaSites2005/Dracunculiasis/eradication.htm.

[22] Donald, R., Ernesto, R., Mark, I. and Sharon, I. (2014) Morbidity and Mortality Weekly Report: Progress toward Global Eradication of Dracunculiasis-January 2013, June 2014. 1050-1054. 63, No. 46. Retrieve form UMUC Data Base on February, 10, 2014.

[23] Mawusi, A. (2009) Countdown to Wipe out Guinea-Worm in Ghana. Bulletin of the World Health Organization, 87, 649-650. http://dx.doi.org/10.2471/BLT.09.010909

[24] Nathaniel, R. (2013) Of Water and Worms: Guinea Worm Re-Emergence in Niger. Journal of Water and Health, 12, 184-195.

[25] Visser, B.J. (2012) Dracunculiasis Eradication: Finishing the Job before Surprises Arise. Asian Pacific Journal of Tropical Medicine, 5, 505-510. http://dx.doi.org/10.1016/S1995-7645(12)60088-1

[26] Tucker, C. (2012) As Eradication of Guinea Worm Nears, Lessons for Public Health: Disease to Be Second Eliminated Globally. The Nation's Health, 42, 1-10.

[27] Awofeso, N. (2013) Towards Global Guinea Worm Eradication in 2015: The Experience of South Sudan. International Journal of Infectious Diseases, 17, E577-E582. http://dx.doi.org/10.1016/j.ijid.2013.03.003

[28] Biswas, G., Sankara, D., Agua-Agum, J. and Maiga, A. (2013) Dracunculiasis (Guinea Worm Disease): Eradication without a Drug or a Vaccine. Philosophical Transactions of the Royal Society B: Biological Sciences, 370, 1-13. http://dx.doi.org/10.1098/rstb.2012.0146

[29] Greenaway, C. (2004) Dracunculiasis (Guinea Worm Disease). Canadian Medical Association Journal, 170, $495-500$.

[30] Esrey, S., Potash, J., Roberts, L. and Shiff, C. (1991) Effects of Improved Water Supply and Sanitation on Ascariasis, Diarrhea, Dracunculiasis, Hookworm Infection, Schistosomiasis, and Trachoma. Bulletin of the World Health Organization, 69, 609-621.

[31] Kurik, K. and Pereira, G. (2006) The Problem of Water Contamination with Dracunculus medinensis in Southern Sudan. Journal of Rural and Tropical Public Health, 5, 49-58.

[32] Molyneux, D. (2006) Dracunculiasis (Guinea Worm Disease) Eradication. In: Molyneux, D., Ed., Control of Human Parasitic Diseases, Academic Press, Amsterdam.

[33] Richards, F., Ruiz-Tiben, E. and Hopkins, D. (2011) Dracunculiasis Eradication and the Legacy of the Smallpox Campaign: What's New and Innovative? What's Old and Principled? Vaccine, 295, D86-D90. http://dx.doi.org/10.1016/j.vaccine.2011.07.115

[34] Callahan, K., Bolton, B., Hopkins, D., Ruiz-Tiben, E., Withers, P., Meagley, K. and Ngondi, J. (2013) Contributions of the Guinea Worm Disease Eradication Campaign toward Achievement of the Millennium Development Goals. PLoS Neglected Tropical Diseases, 7, e2160. http://dx.doi.org/10.1371/journal.pntd.0002160

[35] CDC (n.d.) Renewed Transmission of Dracunculiasis—Chad, 2010.

http://www.cdc.gov/mmwr/preview/mmwrhtml/mm6022a4.htm

[36] WHO (2012) About Guinea-Worm Disease. http://www.who.int/dracunculiasis/disease/disease_more/en/

[37] Medscape (n.d.) Dracunculiasis. http://emedicine.medscape.com/article/997617-treatment 
[38] (2014) Guinea Worm Disease Symptoms, Causes, Treatment-What Are the Signs and Symptoms of Guinea Worm Disease? Medicine Net. http://www.medicinenet.com/guinea_worm_disease/page2.htm

[39] Smith, G., Blum, D., Huttly, S., Okeke, N., Kirkwood, B. and Feachem, R. (2012) Disability from Dracunculiasis: Effect on Mobility. Annals of Tropical Medicine and Parasitology, 83, 151-158.

[40] Centers of Disease Control and Prevention, CDC (2014) Morbidity and Mortality Weekly Report (MMWR): Progress toward Global Eradication of Dracunculiasis_-January 2013-June 2014. MMWR, 63.

\section{Abbreviations}

CDC: Center for Disease Control and Prevention

EU: European Union

GWD: Guinea Worm Disease

ICCDE: International Commission for the Certification of Dracunculiasis Eradication

JAICA: The Japanese International Cooperation Agency

NGWEP: National Guinea Worm Eradication Program

TCC: The Carter Center

UNICEF: United Nations Children's Emergency Fund

WHO: World Health Organization 